\title{
On Deterministic Approximation of Markov Processes by Ordinary Differential Equations
}

\author{
L. I. ROZONOER ${ }^{*}$ \\ Institute of Control Sciences, Moscow, Russia
}

(Received 25 September, 1996)

For a class of Markov processes on the integer multidimensional lattice, it is shown that the evolution of the mean values of some random variables can be approximated by ordinary differential equations. To illustrate the approach, a Markov model of a chemical reaction is considered.

Keywords: Markov processes; convergence; differential equations; chemical kinetics.

\section{A SEQUENCE OF MARKOV PROCESSES}

Consider a sequence of bounded point sets, $G^{N}, N=1,2, \ldots$, which belong to an $l$-dimensional integer lattice, $Z^{l}$, and define a sequence of continuous time Markov processes, $M^{N}$, as follows:

Let $\Delta^{s}=\left(\Delta_{1}^{s}, \ldots, \Delta_{l}^{s}\right), \mathrm{s}=1, \ldots, r$, be a fixed set of $r$ vectors which belong to $Z^{l}$ and $\pi_{s}^{N}(\xi), N=1,2, \ldots$, be a sequence of $r$ functions $(s=1, \ldots, r)$ which are defined for $\xi \in G^{N}$ and which satisfy the conditions

$$
\begin{gathered}
\pi_{s}^{N}(\xi) \geq 0, \quad \text { if } \xi \in G^{N}, \\
\pi_{s}^{N}(\xi)=0, \quad \text { if } \xi \in G^{N}, \xi+\Delta_{s} \notin G^{N} .
\end{gathered}
$$

* Address for correspondence: L. I. Rozonoer, 61 Moraine Street, \#4, Belmont, MA 02178, USA. 
Assume that the transition probability $P^{N}(\eta \mid \xi, \tau)$ from state $\xi$ to state $\eta$ during time $\tau$ satisfies the conditions

$$
\begin{gathered}
P^{N}(\eta \mid \xi, \tau)=0, \text { if } \eta \neq \xi \text { and } \eta \neq \xi+\Delta^{s} \text { for all s, } \\
P^{N}(\eta \mid \xi, \tau)=\pi_{s}^{N}(\xi) \tau+O\left(\tau^{2}\right), \text { if } \xi \in G^{N}, \eta=\xi+\Delta^{s} \text { for some s, } \\
P^{N}(\eta \mid \xi, \tau)=1-\sum_{s=1}^{r} \pi_{s}^{N}(\xi) \tau+O\left(\tau^{2}\right), \text { if } \eta=\xi \in G^{N} .
\end{gathered}
$$

Thus, $\Delta^{1}, \ldots, \Delta^{r}$, are possible jumps and $\pi_{1}^{N}(\xi) \mathrm{dt}, \ldots, \pi_{r}^{N}(\xi) d t$ are the probabilities of the jumps in the infinitesimal time, $d t$. This means that the following equality is satisfied:

$$
\left(\partial P^{N}(\eta \mid \xi, \tau) / \partial \tau\right)_{\tau=0}=\sum_{s=1}^{r}\left[\left(\delta\left(\eta-\xi-\Delta^{s}\right)-\delta(\eta-\xi)\right] \pi_{s}^{N}(\xi),\right.
$$

where $\delta(\zeta)=1$ if $\zeta=0$, and $\delta(\zeta)=0$ if $\zeta \neq 0$.

One can deduce from (3) that the probability of a state $\xi \in G^{N}$ at time $t$, $w^{N}(\xi, t)$, satisfies the equation

$$
\partial w^{N}(\xi, t) / \partial t=\sum_{s=1}^{r}\left(\pi_{s}^{N}\left(\xi-\Delta^{S}\right) w^{N}\left(\xi-\Delta^{s}, t\right)-w^{N}(\xi, t) \pi_{s}^{N}(\xi)\right),
$$

where it is postulated that $w^{N}\left(\xi-\Delta^{s}, t\right)=0$ if $\xi-\Delta^{s} \notin G^{N}$. Taking into account this convention, one can deduce from (4) that

$$
d\left[\sum_{\xi \in G^{N}} w^{N}(\xi, t)\right] / d t=0 .
$$

If a sequence of initial probabilities $w^{N}(\xi, 0)$ is given, the sequence of probabilities $w^{N}(\xi, t)$ is determined by (4). Thus, Markov process $\xi_{t}^{N}$ on the state space $G^{N} \subseteq Z^{l}$ is defined.

For a given function $\phi: R^{l} \rightarrow R^{l}$, let $E_{\phi}^{N}(t), d_{\phi}^{N}(t)$ denote the mean value and the variance of a random variable $\phi\left(\xi_{t}^{N} / N\right)$, that is

$$
\begin{gathered}
E_{\phi}^{N}(T)=\sum_{\xi \in G^{N}} \phi\left((\xi / N) w^{N}(\xi, t),\right. \\
d_{\phi}^{N}(t)=\sum_{\xi \in G^{N}}\left\|\phi(\xi / N)-E_{\phi}^{N}(t)\right\|^{2} w^{N}(\xi, t) .
\end{gathered}
$$


Here $\|\cdots\|$ denotes the Euclidean norm. In the case $\phi(x)=x$ denote

$$
\begin{gathered}
E_{x}^{N}(T)=\sum_{\xi \in G^{N}}(\xi / N) w^{N}(\xi, t), \\
\left.d_{x}^{N}(t)=\sum_{\xi \in G^{N}} \| \xi / N\right)-E_{x}^{N}(t) \|^{2} w^{N}(\xi, t)^{2} .
\end{gathered}
$$

\section{MAIN RESULTS}

Consider the sets $Q^{N}=\left\{x \in R^{l}: x=\xi / N, \xi \in G^{N}\right\}$ and suppose that there exists such a compact set $Q \subset R^{l}$ that $Q^{N} \subseteq Q$ for all N. In addition, suppose that functions $\pi_{s}^{N}(\xi)$ obey the following condition : There exist functions $\mathrm{a}_{s}(x), x \in Q$, and a sequence $\left\{\varepsilon^{N}\right\}, \varepsilon^{N}>0$, such that

$$
\left|\pi_{s}^{N}(\xi)-a_{s}(\xi / N)\right| \leq \varepsilon^{N}, \xi \in G^{N}, \quad \lim _{N \rightarrow \infty} \varepsilon^{N}=0 .
$$

In the following, we assume that all $\mathrm{a}_{s}(x)$ belong at least to $C^{2}$.

Consider the system of differential equations

$$
d x_{i} / d t=\sum_{s=1}^{r} \Delta_{i}^{s} a_{s}(x), \quad i=1, \ldots, l .
$$

Denote by $x\left(x^{\circ}, t\right)$ the solution of the system with an initial condition $x(0)=x^{\circ}$. Our main problem is to demonstrate a close relation, in the limit as $N \rightarrow \infty$, between Markov process $\xi_{t}^{N}$ and the solution of (9). For this reason, we need the following condition:

\section{Condition A}

1. There exists a constant $L$ such that for any pair of points $x^{1} \in Q$ and $x^{2} \in Q$, there exists a smooth curve $\Gamma \subset Q$ with ends in $x^{1}$ and $x^{2}$ and its length, $L\left(x^{1}, x^{2}\right)$, obeys the inequality $L\left(x^{1}, x^{2}\right) \leq L\left\|x^{1}-x^{2}\right\|$;

2. Functions $a_{s}(x)$ belong to $C^{2}$;

3. If $x^{\circ} \in Q$, then $x\left(x^{\circ}, t\right) \in Q$ for all $t>0$;

4. System (9) has an unique equilibrium point $x^{\infty}$, which is an asymptotically stable and is an inner point of $Q$; the convergence, $x\left(x^{\circ}, t\right) \rightarrow x^{\infty}(t \rightarrow \infty)$, is uniform with respect to all $x^{\circ} \in Q$. 
Our main result is contained in Theorem 1 below. There is a similarity between this theorem and the well-known results on the so-called density dependent population processes, see [1]-[4]. General approaches to the convergence of Markov processes based on a semi-group characterization, on stochastic equations and on the martingale theorems were considered in the book [4]. Markov processes for discrete time were considered in [5], [6], [7]. Our approach is not based on the above approaches; it relies on a possibility to introduce " hidden" random variables whose mean values obey - asymptotically - a closed (in the sense that no other variables are involved) system of ordinary differential equations and whose variance is negligible. Such a method is conceptually similar those used in statistical physics. The theorem is applicable to problems of population dynamics, chemical kinetics, and others, allowing the deterministic approximation of the stochastic dynamics.

THEOREM 1 Assume that the sequence of random processes, $M^{N}$, obeys (8) and that Condition A is satisfied. Then the following is true:

1. For any sequence of initial distributions, $w^{N}(\xi, 0)$,

$$
\lim _{N \rightarrow \infty} \varlimsup_{t \rightarrow \infty}\left\|E_{x}^{N}(t)-x^{\infty}\right\|=0, \quad \lim _{N \rightarrow \infty} \varlimsup_{t \rightarrow \infty} d_{x}^{N}(t)=0 ;
$$

2. If for a sequence of initial distributions, $w^{N}(\xi, 0)$, and for some $x^{\circ} \in Q$

$$
\lim _{N \rightarrow \infty}\left\|E_{x}^{N}(0)-x^{\circ}\right\|=0, \quad \lim _{N \rightarrow \infty} d_{x}^{N}(0)=0
$$

then

$$
\lim _{N \rightarrow \infty}\left\|E_{x}^{N}(t)-x\left(x^{\circ}, t\right)\right\|=0, \quad \lim _{N \rightarrow \infty} d_{x}^{N}(t)=0
$$

uniformly for $t \in[0, \infty\}$ and $x^{\circ} \in Q$.

THEOREM 2 Let the conditions of Theorem 1 be satisfied. Then there exists an injection $\phi: Q \rightarrow \phi(Q) \subset R^{l}, y=\phi(x), \phi\left(x^{\infty}\right)=0$, which belongs together with its inverse to the class $C^{1}$, such that

1. For any sequence of initial distributions, $w^{N}(\xi, 0)$,

$$
\lim _{N \rightarrow \infty} \varlimsup_{t \rightarrow \infty}\left\|E_{\phi}^{N}(t)\right\|=0, \quad \lim _{N \rightarrow \infty} \varlimsup_{t \rightarrow \infty} d_{\phi}^{N}(t)=0 ;
$$

2. If for a sequence of initial distributions, $w^{N}(\xi, 0)$,

$$
\left.\lim _{N \rightarrow \infty} \| E_{\phi}^{N}(0)-\phi\left(x^{\circ}, t\right)\right) \|=0, \quad \lim _{N \rightarrow \infty} d_{\phi}^{N}(0)=0
$$


then, uniformly for $t \in[0, \infty)$ and $x^{\circ} \in Q$,

$$
\lim _{N \rightarrow \infty}\left\|E_{\phi}^{N}(t)-\phi\left(x\left(x^{\circ}, t\right)\right)\right\|=0, \quad \lim _{N \rightarrow \infty} d_{\phi}^{N}(t)=0 .
$$

The assertions of the Theorems can be improved at the price of strengthening Condition A.

\section{Condition B}

The items 1)-4) of Condition A are satisfied and, in addition,

5. The linearization matrix of system (9) has eigenvalues $\lambda_{1}, \ldots, \lambda_{l}\left(\operatorname{Re} \lambda_{i}<0\right)$ such that there does not exist a set of natural numbers $m_{1}, \ldots, m_{l}, \Sigma m_{j} \geq 2$, for which the equality $\lambda_{i}=\sum_{j=1}^{l} \lambda_{j} m_{j}$ takes place for some $i=1, \ldots, l$;

6. Functions $a_{s}(x)$ belong to the class $C^{n}$ where $n \geq 2$ and

$$
n \geq \max \left\{\left|\lambda_{1}\right|, \ldots,\left|\lambda_{l}\right|\right\} / \min \left\{\left|\lambda_{1}\right|, \ldots,\left|\lambda_{l}\right|\right\} ;
$$

7. In formula (8), $\varepsilon^{N} \leq b / N$ where $b>0$ is a constant.

The following Theorems $1 \mathrm{~B}$ and $2 \mathrm{~B}$ are analogous to Theorems 1 and 2.

THEOREM 1B Assume that the sequence of random Markov processes, $M^{N}$, obeys conditions (1)-(4), (8) and Condition B is satisfied. Then the following is true:

1. There exist constants, $c_{1}>0, c_{2}>0$, such that uniformly for any sequence of initial distributions

$$
\varlimsup_{t \rightarrow \infty}\left\|E_{x}^{N}(t)-x^{\infty}\right\|<c_{1} / \sqrt{N}, \quad \varlimsup_{t \rightarrow \infty} d_{x}^{N}(t)<c_{2} / N ;
$$

2. For any $b_{1}>0, b_{2}>0$ there exist constants $c_{3}>0, c_{4}>0$ such that if for any sequence of initial distributions, $w^{N}(\xi, 0)$, and for $x^{\circ} \subset Q$

$$
\left\|E_{x}^{N}(0)-x^{\circ}\right\|<b_{1} / \sqrt{N}, \quad d_{x}^{N}(0)<b_{2} / N
$$

then for all $t \in[0, \infty)$

$$
\left|E_{x}^{N}(t)-x\left(x^{\circ}, t\right)\right|<c_{3} / \sqrt{N}, \quad d_{x}^{N}(t)<c_{4} / N .
$$

THEOREM 2B Let the conditions of Theorem 1B be satisfied. Then there exists an injection $\phi: Q \rightarrow \phi(Q) \subset R^{l}, y=\phi(x), \phi\left(x^{\infty}\right)=0$, which belongs together with its inverse to the class $C^{n}$ and such that

1. There exist constants, $\hat{c_{1}}>0, \hat{c_{2}}>0$, such that for any sequence of initial distributions $w^{N}(\xi, 0)$

$$
\varlimsup_{t \rightarrow \infty}\left\|E_{\phi}^{N}(t)\right\|<\hat{c}_{1} / N, \quad \varlimsup_{t \rightarrow \infty} d_{\phi}^{N}(t)<\hat{c}_{2} / N
$$


2. For any $b_{1}>0, b_{2}>0$, there exist constants $\hat{c_{3}}>0, \hat{c_{4}}>0$ such that if for a sequence of initial distributions $w^{N}(\xi, 0)$ with $x^{\circ} \in Q$ $\left\|E_{\phi}^{N}(0)-\phi\left(x^{\circ}\right)\right\|<b_{1} / N^{\mu}, \quad d_{\phi}^{N}(0)<b_{2} / N^{\nu} ; 0<\mu \leq 1,0<v \leq 1$, then for all $t \in[0, \infty)$

$$
\left\|E_{\phi}^{N}(t)-\phi\left(x\left(x^{\circ}, t\right)\right)\right\|<\hat{c}_{3} / N^{\mu}, \quad d_{\phi}^{N}(t)<\hat{c}_{4} / N^{v} .
$$

\section{PROOFS}

\section{Proof of Theorem 2 and Theorem 2B}

The arguments are based on the work of Hartman and Steinberg who strengthened the well-known results of Grobman-Hartman asserting the existence of a mapping that transforms a nonlinear system of differential equations to a linear one [8]. Denote by $A$ the linearization matrix of system (9) at point $x^{\infty}$.

LEMMA 1 Let Condition A hold. Then there exists an injection $\phi: Q \rightarrow \phi(Q) \subset R^{l}, y=\phi(x), \phi\left(x^{\infty}\right)=0$, belonging together with its inverse to the class $C^{1}$, such that on each trajectory, $x(t)$, of the system (9), function $y(t)=\phi(x(t))$ obeys the linear equation

$$
d y / d t=A y \text {. }
$$

LEMMA 1B Let Condition B hold. Then the mapping $y=\phi(x)$, the existence of which is guaranteed by Lemma 1, belongs together with its inverse to the class $C^{n}$.

To prove Lemmas 1 and $1 \mathrm{~B}$, note first that the existence of a mapping, $\hat{\phi}(x)$, which transforms system (9) into (10) in a sufficiently small neighborhood, $E$, of point $x^{\infty}$ is guaranteed by Hartman theorem, if Condition $\mathrm{A}$ is satisfied, and by Steinberg theorem, if Condition B is satisfied [8]. To complete the proof of the lemmas, we have to construct an extension of the mapping $\hat{\phi}(x)$ from $E$ to $Q$. There exist $\hat{t}$ such that $x\left(x^{\circ}, \hat{t}\right) \in E$ for all $x^{\circ} \in Q$, because $x\left(x^{\circ}, t\right)$ converges to $x^{\infty}$ uniformly for $x^{\circ}$. The theorem on dependence of solutions of differential equations on initial data says that the mapping $\Psi\left(x^{\circ}\right)=x\left(x^{\circ}, \hat{t}\right)$ belongs to class $C^{1}$ if Condition $\mathrm{A}$ is satisfied and to the class $C^{n}$ if Condition B is satisfied. The extension, $y=\phi(x)$, is constructed now as $\phi(x)=\exp (-\mathrm{A} \hat{t}) \hat{\phi}(\Psi(x))$. It belongs to the class $C^{1}$ if Condition A holds and to the class $C^{n}$ if Condition B holds. The last step of the 
proof is to show that function $y(t)=\phi\left(x\left(x^{\circ}, t\right)\right)$ can be represented in the form $\exp (A t) \phi\left(x^{\circ}\right)=\exp (A t) y(0)$, i.e., as a solution of equation (10) for all $x^{\circ} \in Q$. We can conclude from the linearization theorem that function $\hat{\phi}(x)$ has such property, i.e., $\hat{\phi}\left(x\left(x^{\circ}, t\right)\right)=\exp (A t) \hat{\phi}\left(x^{\circ}\right)$ if $x^{\circ} \in E$. The stationary property of system (9), and the fact that its solutions form a semi-group of mappings, lead to the equalities $x\left(x\left(x^{\circ}, t\right), \hat{t}\right)=x\left(x\left(x^{\circ}, \hat{t}\right), t\right)$ and $\Psi\left(x\left(x^{\circ}, t\right)\right)=x\left(\Psi\left(x^{\circ}\right)\right.$, $t$ ). Therefore, using the definition of function $\phi(x)$, we have the following chain of the equalities for all $t$ and $x^{\circ} \in Q$ :

$$
\begin{aligned}
\phi\left(x\left(x^{\circ}, t\right)\right) & =\exp (-A \hat{t}) \hat{\phi}\left(\Psi\left(x\left(x^{\circ}, t\right)\right)\right) \\
& =\exp (-A \hat{t}) \hat{\phi}\left(x\left(\Psi\left(x^{\circ}\right), t\right)\right) \\
& =\exp (-A \hat{t}) \exp (A t) \hat{\phi}\left(\Psi\left(x^{\circ}\right)\right) \\
& =\exp (A t) \exp (-A \hat{t}) \hat{\phi}\left(\Psi\left(x^{\circ}\right)\right) \\
& =\exp (A t) \phi\left(x^{\circ}\right) .
\end{aligned}
$$

This implies that

$$
y(t)=\exp (A t) y(0)
$$

The lemmas are proved.

We can conclude from the lemmas that the function $\phi(x)$ obeys the identity

$$
\sum_{i=1}^{l} \partial \phi_{j} / \partial x_{i} \sum_{s=1}^{r} \Delta_{i}^{s} a_{s}(x)=\sum_{k=1}^{l} A_{j k} \phi_{k}
$$

where $\sum_{s=1}^{r} \Delta_{i}^{s} a_{s}(x), i=1, \ldots, l$, are the right sides of the equations (9), and $\sum_{k=1}^{l} A_{j k} \phi_{k}, j=1, \ldots, l$ are the right sides of the equations (10).

Consider the evolution in the time $t$ of the mean values of the random quantity $h\left(\xi_{t}^{N} / N\right)$ using the equation (4). Taking into account (2) and the convention $w^{N}\left(\xi-\Delta^{s}, t\right)=0$, if $x-\Delta^{s} \notin G^{N}$, we come to the equation

$$
d E_{h}^{N} / d t=\sum_{\xi \in G^{N}} \sum_{s=1}^{r}\left[h\left(\xi / N+\Delta^{s} / N\right)-h(\xi / N)\right] \pi_{s}^{N}(\xi) w^{N}(\xi, t),
$$

where $E_{h}^{N}(t)$ is a mean value of the quantity $h\left(\xi_{t}^{N} / N\right)$, and $\Sigma^{\prime}$ stands for a sum over $\xi \in G^{N}$ such that $\xi+\Delta^{s} \in G^{N}$ for some $\overline{\mathrm{S}}$. If $h(x)$ belongs to the class $C^{1}$, then 


$$
h\left(x+\Delta^{s} / N\right)-h(x)=1 / N\left[\sum_{i=1}^{l} \partial h / \partial x_{i} \Delta_{i}^{s}\right]+\alpha^{N}(x),
$$

where $\alpha^{N}(x) \rightarrow 0$ if $N \rightarrow \infty$ uniformly for $x \in Q$. If $h(x)$ belongs to class $C^{2}$, then for $x \in Q$, the inequality $\left|\alpha^{N}(x)\right|<\alpha_{1} / N$ holds, where $\alpha_{1}>0$ is a constant. From (12) and (13), taking into account the boundedness of continuous functions on the compact $Q$, we have

$$
d E_{h}^{N} / d t=E\left\{\sum_{i=1}^{l}\left[\partial h / \partial x_{i} \sum_{s=1}^{r} \Delta_{i}^{s} a_{s}(x)\right]_{x=\xi / N}\right\}+\eta^{N}(t),
$$

where the $E$ is the symbol of the mean value of the expression in the braces in which the substitution $x=\xi / N$ is made, and $\left|\eta^{N}(t)\right| \rightarrow 0$ when $N \rightarrow 0$. Besides, if $\varepsilon^{N} \leq b / N$ in (8), then $\mid \eta^{N}(t) \|<\alpha / N$, where $\alpha$ is a constant.

Applying formula (14) to functions $\phi_{j}$, we obtain:

$$
d E_{\phi_{j}}^{N}(t) / d t=\sum_{k=1}^{l} A_{j k} E_{\phi_{k}}^{N}+f_{j}^{N}(t)
$$

where $E_{\phi j}^{N}$ is a mean value of $\phi_{j}\left(\xi_{t}^{N} / N\right)$ and $f_{j}^{N}(t) \rightarrow 0$ as $N \rightarrow \infty$ uniformly in $t$. Besides, if the $\phi_{j}$ belongs to the class $C^{2}$ and if $\varepsilon^{N} \leq b / N$ in (8) (i.e.,. if the conditions of Theorem $2 \mathrm{~B}$ are satisfied), then

$$
\left|f_{j}^{N}(t)\right|<\alpha / N
$$

where $\alpha$ is some constant.

Analogously, apply the formula (14) to the products $\phi_{j} \phi_{m}$, taking into account (11). After some calculations, we obtain:

$$
d E^{N}\left\{\phi_{j} \phi_{m}\right\} / d t=\sum_{k=1}^{l} A_{j k} E^{N}\left\{\phi_{k} \phi_{m}\right\}+\sum_{k=1}^{l} A_{m k} E\left\{\phi_{k} \phi_{j}\right\}+\phi_{j m}^{N} .
$$

For the correlation matrix $V^{N}$ with the elements $V_{j k}^{N}=E^{N}\left\{\phi_{k} \phi_{j}\right\}-E_{\phi j}^{N} E_{\phi k}^{N}$, we can obtain, using (15), the equation:

$$
d V^{N} / d t=A V^{N}+V^{N} A^{\prime}+F^{N},
$$

where the prime denotes the transpose and $F^{N}$ is a vector-function for which $\left\|F^{N}\right\| \rightarrow 0$ as $N \rightarrow \infty$, uniformly in $t$. Besides, if the $\phi_{j}$ belongs to class $C^{2}$ and if $\varepsilon^{N} \leq b / N$ in (8) (i.e., if the conditions of Theorem 2B are met), then $\left\|F^{N}\right\|<$ const $/ N$.

Note. By definition of the matrix $V^{N}$, we have $\operatorname{Tr} V^{N}=d_{\phi}^{N}$ (cf. formula (6)). 
LEMMA 2 Consider two systems of linear equations:

$$
\begin{gathered}
d y / d t=A y, \\
d z / d t=A z+f(t) .
\end{gathered}
$$

where the A is a Hurwitz matrix, with initial conditions $y(0)=y^{\circ}, z(0)=z^{\circ}$. Then there exist constants $c_{1}>0, c_{2}>0, \mu>0$ such that if $\|f(t)\| \leq \delta,\left\|y^{\circ}-z^{\circ}\right\| \leq \varepsilon$, then

$$
\|y(t)-z(t)\| \leq c_{1} \varepsilon \exp (-\mu t)+c_{2} \delta
$$

For the proof, write down the solutions of the equations in the form:

$$
\begin{gathered}
y(t)=\exp (A t) y^{\circ}, \\
z(t)=\exp (A t) z^{\circ}+\int_{0}^{t} \exp (A(t-\tau)) f(\tau) d \tau .
\end{gathered}
$$

From this we have:

$$
\|y(t)-z(t)\| \leq\|\exp (A t)\|\left\|y^{\circ}-z^{\circ}\right\|+\left\|\int_{0}^{t} \exp (A(t-\tau)) f(\tau) d \tau\right\|,
$$

Taking into account that $\|\exp (\mathrm{At})\| \leq \mathrm{c}^{1} \exp (-\mu t)$, for the Hurwitz matrix $A$, where $c_{1}$ and $\mu$ are some positive constants, and taking into account the inequalities

$$
\|B v\| \leq\|B\|\|v\|, \quad\left\|\int_{0}^{t} \Omega(\tau) v(\tau) d \tau\right\| \leq \int_{0}^{t}\|\Omega(\tau)\|\|v(\tau)\| d \tau,
$$

we obtain the assertion of Lemma 2 (with $c_{2}=c_{1} / \mu$ ).

LEMMA 3 Let a positively definite matrix $V(t)$ obey the equation

$$
d V / d t=A V+V A^{\prime}+F
$$

with the initial conditions $V(0)=V^{\circ}$, where the $A$ is a Hurwitz matrix. Then there exist constants $c^{\prime}>0, c^{\prime \prime}>0, \mu>0$ such that, if $\operatorname{Tr} V^{\circ}<\varepsilon$, and $\|F(t)\|<\delta$, then

$$
\operatorname{Tr} V(t)<c^{\prime} \varepsilon \exp (-\mu t)+c^{\prime \prime} \delta
$$

For the proof, write down the solution of the equation for the $V(t)$ in the form:

$$
V(t)=\exp (A t) V^{\circ} \exp \left(A^{\prime} t\right)+\int_{0}^{t} \exp (A(t-\tau)) F(\tau) \exp \left(A^{\prime}(t-\tau)\right) d \tau
$$

Estimating the norm $\|V(t)\|$ and taking into account that $\|\exp (A t)\| \leq c_{1} \exp (-\mu t)$, where $c_{1}$ and $\mu$ are some constants, we can obtain (analogously to the proof of Lemma 2):

$$
\|V(t)\|<c_{1}^{2} \exp (-2 \mu t)\left\|V^{\circ}\right\|+\delta c_{1}^{2} / 2 \mu .
$$


For a positive-definite matrix, the inequality takes place:

$$
\|V\|<\operatorname{Tr} V^{\circ}<k\|V\|
$$

where $k$ is the dimension of the matrix. This follows from the observation that the norm coincides with the maximal eigenvalue and the trace is equal to the sum of all eigenvalues. Therefore, $\left\|V^{\circ}\right\|<\operatorname{Tr} V^{\circ}<\varepsilon, \operatorname{Tr} V(t)<k\|V(t)\|$, and we have $\operatorname{Tr} V(t)<k c_{1}^{2} \exp (-2 \mu t) \varepsilon+\delta k c_{1}^{2} / 2 \mu$. Lemma 3 is proved.

To complete the proof of Theorem 2, we use Lemma 1, Lemma 2 together with formula (15) and the assertion that $f_{j}^{N}(t) \rightarrow 0$ as $N \rightarrow \infty$ uniformly for $t$, Lemma 3 together with the note after it, formula (17), and the assertion that $\left\|F^{\mathrm{N}}\right\| \rightarrow 0$ as $N \rightarrow \infty$ uniformly for $t$.

To prove Theorem $2 \mathrm{~B}$, use Lemma $1 \mathrm{~B}$, Lemma 2 together with formula (15) and the estimate $\left|f_{j}^{N}(\mathrm{t})\right|<\alpha / N$, Lemma 3 together with the note after formula (17), formula (17) and the estimate $\left\|F^{N}\right\|<$ const/ $N$. Theorems 2 and $2 \mathrm{~B}$ are proved.

Proof of the Theorems 1 and 1B

To prove these Theorems we need the following lemmas.

LEMMA 4 Let $\phi:: Q \rightarrow \phi(Q) \subset R^{l}, y=\phi(x)$ be an injection of a compact $Q$ to a compact $\phi(Q) \subset R^{l}$, which belongs together with its inverse to the class $C^{1}$. Also, let the assumption 1 of Condition A hold. Then there exist constants $\alpha$ and $\beta$ such that for each pair $x^{\prime} \in Q$, and $x^{\prime \prime} \in Q$

$$
\beta\left\|x^{\prime}-x^{\prime \prime}\right\| \leq\left\|\phi\left(x^{\prime}\right)-\phi\left(x^{\prime \prime}\right)\right\| \leq \alpha\left\|x^{\prime}-x^{\prime \prime}\right\| .
$$

Proof Assumption 1) of Condition A says that there exists a curve, $x=x(\lambda), \lambda \in[0,1]$, such that $x(0)=x, x(1)=x "$

and $\int_{0}^{1}\|d x / d \lambda\| d \lambda \leq L \| \int_{0}^{1}(d x / d \lambda) d \lambda \mid$ where the $L$ is a constant. Denoting $y(\lambda)=\phi(x(\lambda))$, we have $d y / d \lambda=\Phi(x(\lambda)) d x / d \lambda$, where matrix $\Phi(x)$, with the elements $\Phi_{k j}=\partial \phi_{k} / \partial x_{j}$ is non-singular and continuous with respect to $x \in Q$. Its elements are boounded on the compact, and therefore there exist positive constants $\gamma_{1}, \gamma_{2}$ such that $\gamma_{1} \leq\|\Phi(x)\| \leq \gamma_{2}$. Therefore, we have:

$$
\begin{gathered}
\left\|\phi\left(x^{\prime}\right)-\phi\left(x^{\prime \prime}\right)\right\|=\left\|\int_{0}^{1}(d y / d \lambda) d \lambda\right\| \leq \int_{0}^{1}\|d y / d \lambda\| d \lambda \leq \\
\gamma_{2} L\left\|\int_{0}^{1}(d x / d \lambda) d \lambda\right\|=\gamma_{2} L\left\|x^{\prime}-x^{\prime \prime}\right\| .
\end{gathered}
$$

Thus, the right hand side of the required inequality is proved. The left side is proved analogeously by considering the inverse mapping. Lemma 4 is proved. 
LEMMA 5 Let $\phi: Q \rightarrow \phi(Q) \subset R^{l}, y=\phi(x)$ be a mapping which obeys the inequality

$$
\beta\left\|x^{\prime}-x^{\prime \prime}\right\| \leq\left\|\phi\left(x^{\prime}\right)-\phi\left(x^{\prime \prime}\right)\right\| \leq \alpha\left\|x^{\prime}-x^{\prime \prime}\right\|
$$

and $\mu(x)$ be a measure with the support $Q$. Then for any $x^{1} \in Q$ the following inequalities hold:

$$
\begin{gathered}
d_{x} \leq 1 / \beta^{2}\left(d_{\phi}+\left\|E_{\phi}-\phi\left(x^{1}\right)\right\|^{2}\right), \\
\left\|E_{x}-x^{1}\right\| \leq(1 / \beta)\left(d_{\phi}+\left\|E_{\phi}-\phi\left(x^{1}\right)\right\|^{2}\right)^{1 / 2} \\
d_{\phi} \leq \alpha^{2}\left(d_{x}+\left\|E_{x}-x^{1}\right\|^{2}\right) \\
\left\|E_{\phi}-\phi\left(x^{1}\right)\right\| \leq \alpha\left(d_{x}+\left\|E_{x}-x^{1}\right\|^{2}\right)^{1 / 2} .
\end{gathered}
$$

Here $E_{x}, \mathrm{E}_{\phi}, d_{x}, d_{\phi}$ denote the mean values and variances of the random quantities $x$ and $\phi(x)$ with respect to the measure $\mu(x)$ :

$$
E_{\phi}=\int_{Q} \phi(x) d \mu(x), \quad d_{\phi}=\int_{Q}\left\|\phi(x)-E_{\phi}\right\|^{2} d \mu(x) .
$$

Proof Consider the quantity:

$$
E\left\{\left\|\phi(x)-\phi\left(x^{1}\right)\right\|^{2}\right\}=\int_{Q}\left\|\phi(x)-\phi\left(x^{1}\right)\right\|^{2} d \mu(x),
$$

and estimate it, using the inequality in the Lemma condition. Besides, taking into account that

$$
E\left\{\left\|\phi(x)-\phi\left(x^{1}\right)\right\|^{2}\right\}=E\left\{\left\|\phi(x)-E_{\phi}\right\|^{2}\right\}+\left\|E_{\phi}-\phi\left(x^{1}\right)\right\|^{2},
$$

one can come to the inequality

$$
\beta^{2}\left(d_{x}+\left\|E_{x}-x^{1}\right\|^{2}\right) \leq\left(d_{\phi}+\left\|E_{\phi}-\phi\left(x^{1}\right)\right\|^{2} \leq \alpha^{2}\left(d_{x}+\left\|E_{x}-x^{1}\right\|^{2}\right),\right.
$$

which gives the assertion of the Lemma.

For the proof of Theorems 1 and 1B, take $\phi(x)$ from Theorems 2 and $2 B$ and put $x^{1}=x(t)$ in Lemma 5 and choose measure $\mu(x)$ corresponding to the distribution $w^{N}(\xi, t)$ with $x=\xi / N$. Then from the inequalities in Lemma 5 we have:

$$
\begin{gathered}
d_{x}^{N}(t) \leq 1 / \beta^{2}\left(d_{\phi}^{N}(t)+\left\|E_{\phi}^{N}(t)-\phi(x(t))\right\|^{2}\right) \\
\left\|E_{x}^{N}(t)-x(t)\right\| \leq 1 / \beta\left(d_{\phi}^{N}(t)+\left\|E_{\phi}^{N}(t)-\phi(x(t))\right\|^{2}\right)^{1 / 2} \\
d_{\phi}^{N}(t) \leq \alpha^{2}\left(d_{x}^{N}(t)+\left\|E_{x}^{N}(t)-x(t)\right\|^{2}\right) \\
\left\|E_{\phi}^{N}(t)-\phi(x(t))\right\| \leq \alpha\left(d_{x}^{N}(t)+\left\|E_{x}^{N}(t)-x(t)\right\|^{2}\right)^{1 / 2}
\end{gathered}
$$


Putting $x^{1}=x^{\circ}, t=0$ in Lemma 5, we obtain the inequalities:

$$
\begin{aligned}
\left\|E_{x}^{N}(0)-x^{\circ}\right\| & \leq 1 / \beta\left(d_{\phi}^{N}(0)+\left\|E_{\phi}^{N}(0)-\phi\left(x^{\circ}\right)\right\|^{2}\right)^{1 / 2} \\
\left\|d_{\phi}^{N}(0)\right\| & \leq \alpha\left(d_{x}^{N}(0)+\left\|E_{x}^{N}(0)-x^{\circ}\right\|^{2}\right)^{1 / 2} \\
d_{x}^{N}(0) & \leq 1 / \beta_{2}\left(d_{\phi}^{N}(0)+\left\|E_{\phi}^{N}(0)\right\|^{2}\right) \\
\left\|E_{\phi}^{N}(0)\right\| & \leq \alpha\left(d_{x}^{N}(0)+\left\|E_{x}^{N}(0)-x^{\circ}\right\|^{2}\right)^{1 / 2}
\end{aligned}
$$

Inequalities (18)-(25) give the possibility to transform the conditions and the assertions of Theorems 2 and $2 \mathrm{~B}$ to the conditions and assertions of Theorems 1 and $1 \mathrm{~B}$ and vice versa. Now Theorems 1 and $1 \mathrm{~B}$ become corollaries of Theorems 2 and $2 \mathrm{~B}$ (one has to put $\mu=1, v=1 / 2$ in Theorem 2B). For example, let us prove Theorem 1 from Theorem 2 . If the assertion 1) of Theorem 2 is true, then $\left(d_{\phi}^{N}(t)+\| E_{\phi}^{N}(t)-\left.\phi(\mathrm{x}(t))\right|^{2}\right) \rightarrow 0$ as $\mathrm{t} \rightarrow \infty$. Inequalities (18) and (19) then lead directly to assertion 1) of Theorem 1 . If the conditions $\left(E_{x}^{N}(0)-x^{\circ}\right) \rightarrow 0$ and $d_{x}^{N}(0) \rightarrow 0$ as $N \rightarrow \infty$ are satisfied, then inequalities (22), (23) guarantee that $\left\|E_{\phi}^{N}(0)-\phi\left(\mathrm{x}^{\circ}\right)\right\| \rightarrow 0$ and $\left\|d_{\phi}^{N}(0)\right\| \rightarrow 0$ as $N \rightarrow \infty$. Then assertion 2$)$ of Theorem 2 gives that $\| E_{\phi}^{N}(t)-\phi\left(x(t) \| \rightarrow 0\right.$ and $d_{\phi}^{N}(t) \rightarrow 0$ as $N \rightarrow \infty$. The inequalities (18) and (19) then lead directly to assertion 2) of Theorem 1.

\section{AN EXAMPLE: A MARKOV MODEL OF THE CHEMICAL KINETICS AND ITS ASYMPTOTIC DESCRIPTION BY ORDINARY DIFFERENTIAL EQUATIONS}

A Markov model of chemical kinetics gives an example of a Markovian process for which an asymptotic description by ordinary differential equations is possible (see, for example, [4] [9]). Consider some reversible chemical reactions taking place in a volume. The reversibility of the reactions means that together with a direct reaction, $\sum_{j=1}^{m} \alpha_{j} A_{j} \rightarrow \sum_{j=1}^{m} \beta_{j} A_{j}$ the inverse reaction, $\sum_{j=1}^{m} \beta_{j} A_{j} \rightarrow \sum_{j=1}^{m} \alpha_{j} A_{j}$ takes place. Here $A_{j}$ is the symbol of a $j$-th substance, $\alpha_{j}, \beta_{j}$ are stoichiometic coefficients $(j=1, \ldots, m)$. For an $i$-th reaction, the stoichiometric coefficients are supplied by andex $i$ : 
$\alpha_{i j}, \beta_{i j}$. Consider first one direct (irreversible) reaction $\sum_{j=1}^{m} \alpha_{j} A_{j} \rightarrow \sum_{j=1}^{m} \beta_{j} A_{j}$. Suppose that the probability of a single act of the reaction per time in a unit volume is proportional to a probability of the event that all molecules of the reacting species come together in volume $\mathrm{V}^{*}$. Denote by $n_{j}$ a number of molecules $j$-th substance. The probability for $\alpha_{j}$ molecules to come together into the volume, $V^{*}$, is set equal to

$$
P_{j}=n_{j}\left(n_{j}-1\right) \cdots\left(n_{j}-\alpha_{j}+1\right)\left(V^{*} / V\right)^{\alpha_{j}},
$$

where the factor $n_{j}\left(n_{j}-1\right) \ldots\left(n_{j}-\alpha_{j}+1\right)$ is equal to the number of ways to choose $\alpha_{j}$ elements from the $n_{j}$ elements. Supposing the independence of the molecules, the probability, $P$, of a reaction act is proportional to the product of probabilities $P=\prod_{j=1}^{m} P_{j}$. Therefore, the probability of one reaction act in all volume per time is equal to

$$
k V P=k V \prod_{j=1}^{m} n_{j}\left(n_{j}-1\right) \cdots\left(n_{j}-\alpha_{j}+1\right)\left(V^{*} / V\right)^{\alpha_{j}},
$$

where $\mathrm{k}$ is a coefficient. The numbers $n_{\mathrm{f}}(t)$ are the variables of a Markov process, but the process is not obviously ergodic because $n_{j}(t)$ depend on the initial data. Introduce in the usual way a coordinate of the reaction, $\xi(t)$, i.e., a number of the reaction acts up to time $t$. Then $n_{j}(t)=n_{j}(0)+\gamma_{j} \xi(t)$, where $\gamma_{j}=\beta_{j}-\alpha_{j}$. Therefore, it is more convenient to consider random process $\xi(t)$ and to express from the beginning $n_{j}(t)$ through $\xi(\mathrm{t})$. If $l$ reactions take place, it is convenient to introduce coordinates of the each, $\xi_{i}(t), i=1, \ldots, l$. When the direct reaction takes place, $\xi_{i}(t)$ increases by 1 , and for an inverse reaction $\xi_{i}(t)$ decreases by 1 . Acts of reactions are assumed to be independent. The numbers of molecules of each species are given by

$$
n_{j}(t)=n_{j}(0)+\sum_{i=1}^{l} \gamma_{i j} \xi_{i}(t), \quad \gamma_{i j}=\beta_{i j}-\alpha_{i j} .
$$

Variables $\xi_{i}(t)$ form a Markov process on the lattice $Z^{l}$ of the kind which was defined in Section 1. Each reversible reaction corresponds to two vectors from the set $\Delta^{s}(s=1, \ldots, 2 l)$ : a direct reaction corresponds to the odd $s$, its inverse to the even one. Components of the vector $\Delta^{s}$ can be written down as

$$
\Delta_{i}^{s}=(-1)^{s+1}\left(\delta_{s(2 i-1)}-\delta_{s(2 i)}\right), \quad s=1, \ldots, 2 l, \quad i=1, \ldots, l,
$$


where $\delta_{k j}$ is the Kronecker's symbol. This formula shows that the coordinate $\xi_{i}(t)$ increased by the direct reaction, and decreased by the inverse one.

Transition probabilities must be expressed through $\xi_{i}$. It can be made by the same suppositions which lead to formula (26). A sequence $M^{N}$ is the corresponding sequence of reactions with large volume and numbers of molecules. Let $V=N V^{*}$. For an odd $s=2 i-1$ (the direct reaction), $\alpha_{i j}$ must be substituted instead of $\alpha_{j}$. For an even $s=2 i$ (the inverse reaction), $\beta_{i j}$ must be substituted instead of $\alpha_{j}$. If we put $V=N V^{*}$ then:

$$
\begin{aligned}
\pi_{2 i-1}^{N} & =k_{2 i-1} V^{* N} \prod_{j=1}^{m} n_{j}\left(n_{j}-1\right) \cdots\left(n_{j}-\alpha_{i j}+1\right)(1 / N)^{\alpha_{i j}}, \\
\pi_{2 i}^{N} & =k_{2 i} V^{* N} \prod_{j=1}^{m} n_{j}\left(n_{j}-1\right) \cdots\left(n_{j}-\beta_{i j}+1\right)(1 / N)^{\beta_{i j}} .
\end{aligned}
$$

In (29), (30) numbers $n_{j}$ must be expressed through $\xi_{i}$ with help of (27). Denoting $z_{j}=n_{j} / N, x_{i}=\xi_{i} / N$ and putting $n_{j}(0) / N=v_{j}^{\circ}$, we have from (27):

$$
z_{j}(t)=v_{j}^{\circ}+\sum_{i=1}^{l} \gamma_{i j} x_{i}(t)
$$

where $\gamma_{i j}=\beta_{i j}-\alpha_{i j}$ The system of inequalities

$$
v_{j}^{\circ}+\sum_{i=1}^{l} \gamma_{i j} x_{i} \geq 0
$$

defines a set $Q$ from Section 2 of the paper.

Taking into account (29) and (30), for functions $\mathrm{a}_{s}(x)$ from Section 1 (formula (8)), we have

$$
a_{2 i-1}(x)=k_{i}^{+} \prod_{j=1}^{m}\left(z_{j}\right)^{\alpha_{i j}}, \quad a_{2 i}(x)=k_{i}^{-} \prod_{j=1}^{m}\left(z_{j}\right)^{\beta_{i j}}
$$

where $z_{j}$ must be expressed through $x_{i}$, and $k_{i}^{+}=k_{2 i-1} V^{*}, k_{i}^{-}=k_{2 i} V^{*}$ are the constants of direct and inverse reactions of type $i$. Besides, $\left|\varepsilon^{\mathrm{N}}\right| \leq$ const/ $N$. Taking into account (28) we can write down the system of differential equations, (9), from Section 2, in the form:

$$
d x_{i} / d t=\left[k_{i}^{+} \prod_{j=1}^{m}\left(z_{j}\right)^{\alpha_{i j}}-k_{i}^{-} \prod_{j=1}^{m}\left(z_{j}\right)^{\beta_{i j}}\right]
$$

where again $z_{j}$ must be expressed through $x_{i}$ with help of (31). 
Equations (34) together with (31) are the phenomenological equations of chemical kinetics based on the law of action masses. If there exists a solution of equations,

$$
\sum_{j=1}^{m} \gamma_{i j} R_{j}=\ln \left(k_{i}^{+} / k_{i}^{-}\right)
$$

then there exists an equilibrium point, i.e. the system of equations

$$
k_{i}^{+} \prod_{j=1}^{m}\left(z_{j}\right)^{\alpha_{i j}}=k_{i}^{-} \prod_{j=1}^{m}\left(z_{j}\right)^{\beta_{i j}}, \quad z_{j}(t)=v_{j}^{\circ}+\sum_{i=1}^{l} \gamma_{i j} x_{i}(t)
$$

has a solution $x^{\infty}$, and, moreover point $x^{\infty}$ is an asymptotically stable equilibrium point of (34) (see, for example [10]). By the way, point $x^{\infty}$ is the maximum point of the function $S(x)=-\sum_{j=1}^{m} z_{j}\left(\ln z_{j}-1\right)+\sum_{j=1}^{m} R_{j} z_{j}$ (where $z_{j}$ must be expressed through $x_{i}$ ) on a linear manifold which is determined by coefficients $\alpha_{i j}, \beta_{i j}$. Function $S(x)$ is the Liapunov function for (34), see [10]. Thus, if a solution of equation (35) exists, then all conditions of Theorem 1 are fulfilled, and the phenomenological equations provide an adequate approximation (in the sense of Theorem 1) of random chemical reactions.

\section{CONCLUDING REMARKS}

The results of the paper demonstrate that Grobman-Hartman mappings $(\phi(x)$ above) play an important role in deterministic approximation of Markov processes considered. An interesting future direction is the consideration of their role in stochastic asymptotic approximations. To this point, a comparison of Theorems 2 and $2 \mathrm{~B}$ leads to the idea of a connection between fluctuation amplitude and a tangential property (smoothness) of Grobman-Hartman mappings. Another direction is the consideration of the mappings for phenomenological equations of chemical kinetics.

\section{Acknowledgements}

The author is grateful to Professor B. Pittel for help in preparation of the paper for publication. 


\section{References}

[1] T. G. Kurtz, "Solutions of ordinary differential equation as limits of pure jump Markov processes," Journal of Applied Probability, vol. 7, No. 1, 1970.

[2] T. G. Kurtz, "Limit theorems for sequences of jump Markov processes approximating ordinary differential equations," Journal of Applied Probability, vol. 8 No. 2, 1971.

[3] T. G. Kurtz, "Strong approximation theorems for density dependent Markov chains," Stoch. Process. Appl., vol. 6, pp. 682-715, 1980.

[4] S. N. Ethier and T. G. Kurtz, Markov processes. Characterization and convergence. Wiley, NY, 1986.

[5] S. M. Meerkov, "Simplified description of slow Markov walks, Part 1," Automation and Remote Control, vol.33, pp. 404-414, 1972.

[6] J. T. Lim, S. M. Meerkov and T. Zheng, "Simplified description of slow-in-the-average Markov walks," Journal of Mathematical Analysis and Applications, vol. 158, No. 2, July, 1991.

[7] L. Ljung, "Analysis of recursive stochastic algorithms," IEEE Transactions on Automatic Control, vol. AC-22, No. 4, 1977.

[8] P. Hartman, The theory of ordinary differential equations, Wiley, NY, 1964.

[9] Mc Quarrie, "Stochastic approach to chemical kinetics," Journal of Applied Probability, vol. 4, No. 3, 1967.

[10] V.N.Orlov and L. I. Rozonoer, "The macrodynamics of open systems and the variational principle of local potential," Journal of the Franklin Institute, vol. 318, No. 5, 1984. 


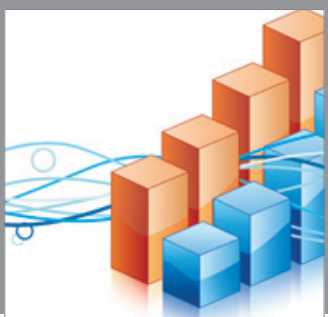

Advances in

Operations Research

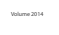

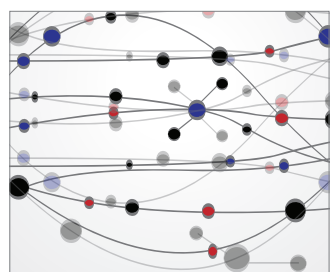

\section{The Scientific} World Journal
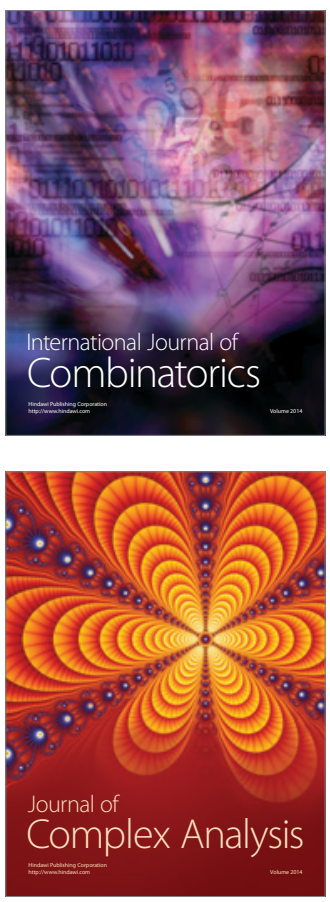

International Journal of

Mathematics and

Mathematical

Sciences
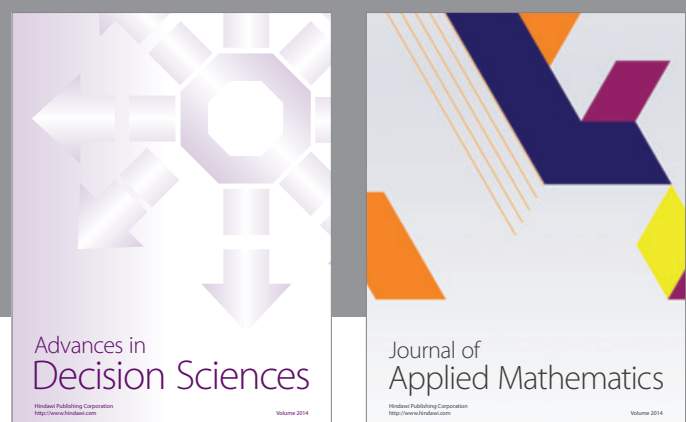

Journal of

Applied Mathematics
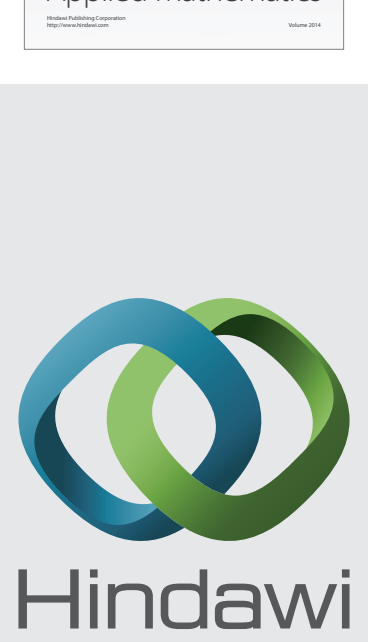

Submit your manuscripts at http://www.hindawi.com
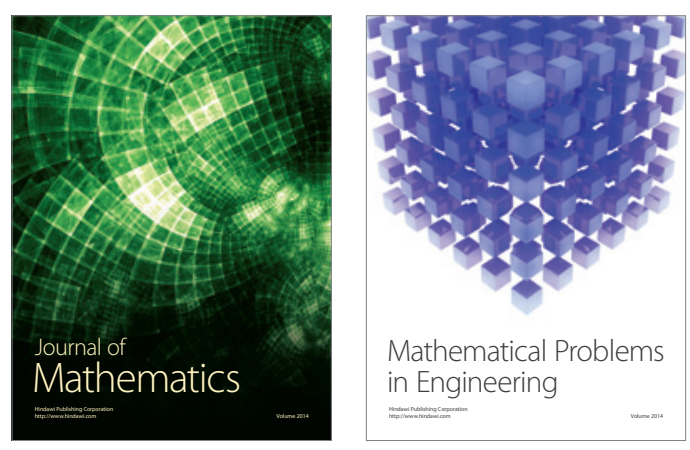

Mathematical Problems in Engineering
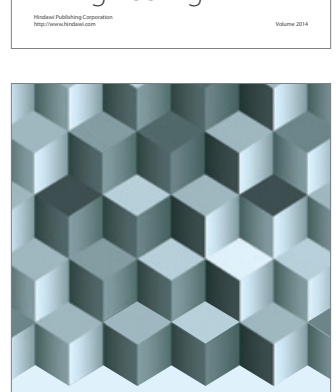

Journal of

Function Spaces
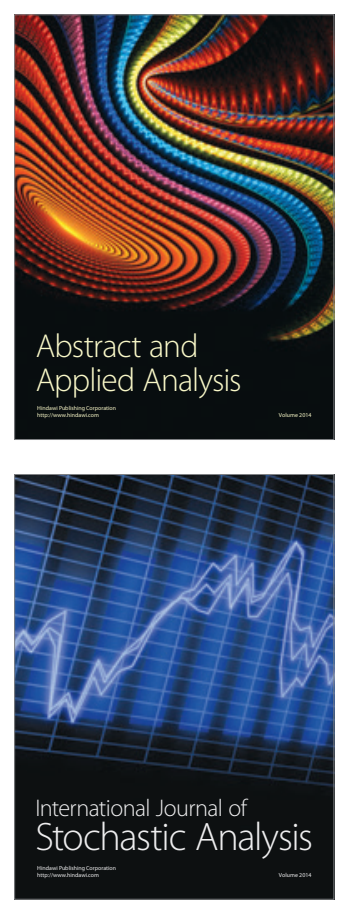

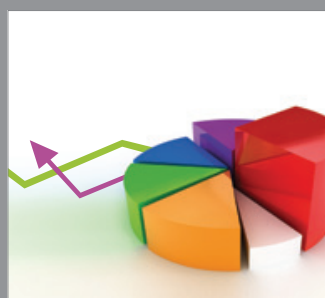

ournal of

Probability and Statistics

Promensencen
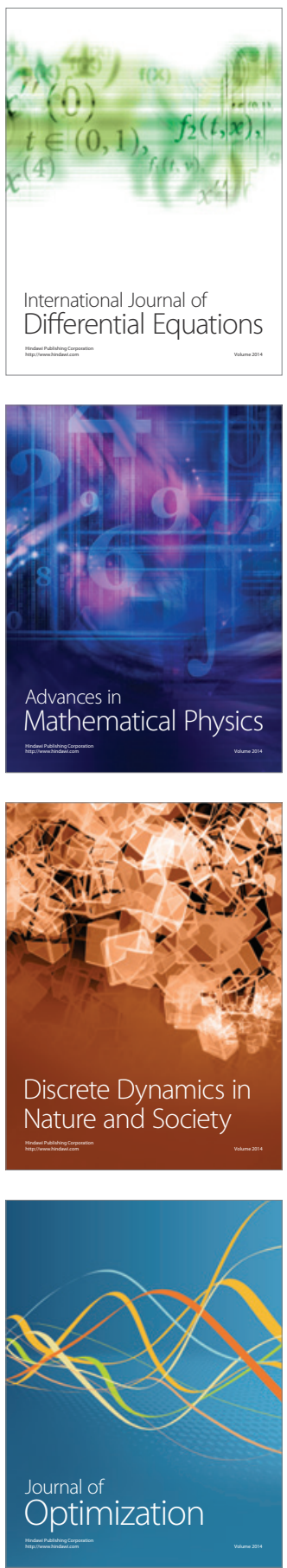